\title{
Estudo comparativo das complica- ções vasculares arteriais e billares no transplante hepático pediátrico com doador vivo e cadavérico
}

\section{Comparative study of arterial and biliary complications in pediatric liver transplantation with living and cadaveric donors}

\author{
Bruna N. Achar'; Niklas S. Campos²; Renata N. Achar³; Eduardo Achar ${ }^{4}$, Marcelo Augusto F. Ribeiro Junior ${ }^{5}$
}

\begin{abstract}
RESUMO:
Introdução: O transplante de fígado melhorar significativamente a taxa de sobrevivência de crianças e adolescentes com doença hepática terminal.Os pacientes, uma vez que tinha um prognóstico fatal, hoje podem ser submetidos a transplante de fígado (TF), com taxas de sobrevida de aproximadamente $90 \%$ em um ano. A atresia biliar é a principal indicação para a população pediátrica e para aqueles sofreram uma portoenterostomia e não obtiveram bons resultados com a mesma, representando mais de $50 \%$ das crianças menores de 2 anos na Europa e nos EUA. O transplante representa a única opção restante de sobrevivência com um bom benefício terapêutico. Os avanços nos cuidados cirúrgicos e clínicos, levaram a melhoria das técnicas para aumentar a sobrevida dos pacientes submetidos a transplante de fígado. Porém, a hemorragia e as complicações biliares representam o tendão de Aquiles do desenvolvimento deste processo.Além disso, a escassez de órgãos para a população pediátrica motiva o desenvolvimento de novas modalidades de enxertos de fígado, como a redução do enxerto de fígado, o transplante de fígado dividido, e mais recentemente o de doadores vivos. As complicações arteriais e biliares continuam a ser uma importante causa de morbidade, mortalidade e perda do enxerto após o transplante. Objetivo: Avaliar as complicações mais frequentes relacionadas às anastomoses biliares e arteriais do transplante hepático pediátrico com doadores vivos e com doadores cadavéricos. Métodos: Pesquisa no Pubmed e Lilacs para reunir dados de complicações hepáticas relacionadas com transplante pediátrico entre 1999 e 2009. Resultados: Neste estudo de 1485 transplantes de fígado utilizando doadores cadavéricos e 505 utilizando dadores vivos, os dados foram coletados e analisados estatisticamente. Ao analisar as complicações arteriais e biliares no transplante hepático pediátrico em intervivos utilizando enxertos, as taxas de fístula biliar foram de 14,7\%, 3,1\%, e de estenoses biliares e tromboses arteriais de 3,3\%. Ao analisar os dados de doadores cadavéricos foi observado que as taxas de trombose arterial foram de 5,7\%,3,7\% de estenoses biliares, e 2,5\% de fístulas biliares. Os dados foram analisados e comparados entre os dois grupos de doadores vivos e cadavéricos, e os resultados foram: fístula biliar $(P=0,0486)^{*}$, estenose biliar $(P=0,5167)$ e trombose da artéria hepática $(P=$ 0,6752). Conclusão: A fístula e a estenose representam as complicações biliares mais frequentes, especialmente na vigência do uso de doadores vivos. Por outro lado, as complicações arteriais mais frequentes acontecem no grupo submetido a enxertos de doadores cadavéricos.
\end{abstract}

Palavras-chave: Transplante de Fígado. Pediatria. Transplante/complicações. Doadores Vivos. Doadores cadavéricos. Doação Dirigida de Tecido. Cadaver.

1. Acadêmica de Medicina Universidade Cidade de São Paulo (UNICID). 2. Acadêmico de Medicina Universidade Metropolitana de Santos. 3. Acadêmica de Medicina Universidade Lusiadas. 4,5. Docente, Habilidades cirúrgicas, Departamento de Medicina, UNICID, 5. Docente de Cirurgia Geral, Coordenador do Núcleo de Clinica Cirúrgica e Coordenador do Curso de Medicina da Universidade de Santo Amato (UNISA)
Correspondência: Universidade Cidade de São Paulo Rua Cesário Galeno, 448/475 - Tatuapé 03071-000 - São Paulo/SP bruna.achar@gmail.com

Artigo recebido em 07/06/2011 Aprovado para publicação em 15/07/2011 


\section{Introdução}

O transplante de fígado melhorou sobremaneira a taxa de sobrevida das crianças e adolescentes com doença hepática terminal. Os pacientes que outrora tinham prognóstico fatal, na atualidade, são submetidos ao transplante hepático (TH), com taxas de sobrevida de aproximadamente $90 \%$ no primeiro ano. ${ }^{1,2}$

O transplante de fígado está indicado em crianças com falência hepática aguda e crônica, doença hepática metabólica hereditária e tumores hepáticos não ressecáveis do tipo hepatocarcinomas fibrolamelares. ${ }^{3}$ Além disso, existem outros indicadores da necessidade de transplante tais como: síndromes colestáticas, prurido e/ou ascite intratáveis clinicamente; hipertensão portal com sangramento de varizes sem resposta ao tratamento; episódios múltiplos de colangite ou episódios de peritonite bacteriana espontânea; síntese hepática progressivamente deficiente; repercussão no crescimento pondo-estatural e encefalopatia hepática. ${ }^{2,4} \mathrm{~A}$ atresia das vias biliares é a principal indicação na faixa etária pediátrica. A falência hepática fulminante secundária a hepatites virais ou intoxicação é indicada, para aqueles pelos quais passaram por uma portoenterostomia e não obtiveram bons resultados com a mesma, que representam mais de $50 \%$ de crianças transplantadas com idade inferior a 2 anos na Europa e nos EUA. ${ }^{2,4,5,6}$

Avanços em cuidados clínicos e em técnica cirúrgica permitiram melhora na sobrevida dos pacientes submetidos a transplante hepático ${ }^{7}$, porém ainda hoje as complicações arteriais e biliares representam o tendão de Aquiles no desenvolvimento desse procedimento. Além disto, a escassez de órgãos para a população pediátrica faz com que novas modalidades de transplante sejam realizadas, como o transplante com enxerto reduzido, o enxerto bipartido ou split-liver além do transplante com doador vivo (THDV).

O conhecimento anatômico e suas peculiaridades tanto arteriais como biliares apresentam grande relevância para o resultado final do transplante na população pediátrica da realização de tal procedimento, portanto, apesar da melhora dos resultados, as complicações vasculares arteriais e biliares permanecem como causa importante de morbidade, mortalidade e perda de enxerto pós-transplante. ${ }^{8}$

\section{Método}

Levantamento de dados nas bases LILACS, Pubmed, Scielo e Medline buscando reunir informa- ções sobre as principais complicações vasculares arteriais e dos ductos biliares pós-cirúrgicas no período de dez anos (1999 - 2009).

\section{Resultados}

Foram analisados 1485 casos de transplante com doador cadáver (TCDC) e 505 casos com doador vivo (TCDV). Esses casos foram coletados em estudos multicêntricos de diversas instituições que realizam transplante pediátrico.

As principais complicações vasculares arteriais e biliares no transplante hepático pediátrico com doador vivo foram, em ordem decrescente, fístula biliar (representando 14,7\% dos casos), trombose da artéria hepática (com $3,3 \%$ dos casos), seguido de estenose biliar $(3,1 \%)$. Já com doadores cadavéricos foram observadas que a fístula biliar $(3,7 \%$ dos casos), estenose biliar (com 2,5\%) e a trombose da artéria hepática $(5,7 \%$ dos casos) foram as principais complicações registradas. ${ }^{9-15}$

\section{Tabela 1}

Frequência (\%) das complicações vasculares arteriais e biliares no transplante hepático pediátrico com doador vivo

\begin{tabular}{lcr}
\hline Complicações & $\mathrm{N}^{\mathrm{o}}$ de casos & $(\%)$ \\
\hline Fistula Biliar & 74 & 14,7 \\
Estenose Biliar & 16 & 3,1 \\
Trombose de Artéria Hepática & 17 & 3,3 \\
\hline
\end{tabular}

\section{Tabela 2}

Frequência (\%) das complicações vasculares arteriais e biliares no transplante hepático pediátrico com doador cadavérico.

\begin{tabular}{lcc}
\hline Complicações & $\mathrm{N}^{\mathrm{o}}$ de casos & $(\%)$ \\
\hline Fistula Biliar & 55 & 3,7 \\
Estenose Biliar & 37 & 2,5 \\
Trombose de Artéria Hepática & 85 & 5,7 \\
\hline
\end{tabular}

A porcentagem de complicações biliares em crianças teve um total de $17,8 \%$ no grupo transplante com doador vivo (THDV) sendo as fistulas responsáveis por $14,7 \%$ dos casos, e a estenoses $3,1 \%$. No grupo transplante doador cadáver (THDC) as fístulas e estenoses representaram $3,7 \%$ e $2,5 \%$, respectiva- 
mente. A trombose da artéria hepática ocorreu respectivamente em 5,7\% dos THDC e 3,3\% dos THDV.

Foram analisados comparativamente os grupos com doadores vivos e cadavéricos quanto às variáveis estudadas aplicando-se o teste $T$ - student onde observou-se para fistula biliar $\mathrm{P}=0,0486$; sendo a única variável com significância estatística. Quando se avaliou estenose biliar e trombose de artéria, foram obti$\operatorname{dos} \mathrm{P}=0,5167$ e $\mathrm{P}=0,6752$ respectivamente, ou seja não significantes.

\section{Discussão}

O TH pediátrico é atualmente tratamento de eleição para crianças que sofrem de insuficiência hepática terminal em países desenvolvidos e subdesenvolvidos. As indicações para transplante incluem insuficiência hepática aguda e crônica, doença hepática metabólica, hereditária e tumores hepáticos não ressecáveis. ${ }^{16,17}$

O primeiro transplante inter vivos foi realizado por RAIA et AL. ${ }^{3,18}$, em 1989, e em 1990, STRONG et al. ${ }^{3,19}$ realizaram o primeiro transplante hepático inter vivos com êxito. HASHIKURA et al. ${ }^{3,20}$, em 1994, realizaram o primeiro transplante inter vivos entre adultos, utilizando o lobo esquerdo do fígado. Inicialmente, essa modalidade de transplante era indicada para crianças e adultos em condições graves e em países onde o número de doadores cadáveres era pequeno ou inexistente. Em nosso meio, a técnica cirúrgica nos transplantes com doadores vivos foram divulgadas por CARONE et al. ${ }^{21}$, em $1997^{3}$.

Números expressivos de complicações vasculares arteriais e biliares podem ser atribuídos a falhas no reconhecimento de variações anatômicas. ${ }^{25}$ Diferenças anatômicas existem entre as vias biliares dos dois lobos hepáticos ${ }^{3,22}$, o que dificulta ainda mais os pré-cuidados no momento do transplante.

Algo importante a ser destacado é que o prognóstico é melhor, ou seja, o risco de complicações é menor (10\%) em crianças do que em adultos, devido a uma parte das crianças terem a capacidade de desenvolver uma rede arterial efetiva ${ }^{7}$, sendo que não necessitará de retransplante, o que resultará em prognósticos melhores. ${ }^{17}$

Em um estudo de sobrevida de pós-transplantes foram criadas porcentagens com base em pacientes transplantados na faixa etária de 0-18 anos, o qual não demonstra especificidade quanto ao tipo de doador. Nesse estudo foram avaliados 84 crianças e ado- lescentes inscritos em lista de transplante hepático. Destes, 40 pacientes foram submetidos a 42 transplantes (dois retransplante), 26 faleceram enquanto aguardavam o transplante e 18 pacientes aguardavam o procedimento ao final da avaliação para esse estudo. ${ }^{(6)}$ Treze $(32,5 \%)$ dos 40 transplantados morreram, com idade na ocasião do óbito que variou de 1,8 a 13,6 anos (mediana de 6,7 anos). Os óbitos ocorreram entre 0 e 204 dias pós-transplante, mediana de 7 dias (IQ25-75\% $)^{4-12} \mathrm{Na}$ análise dos 40 pacientes transplantados, a probabilidade de sobrevida com 180 dias foi de $70 \%$, e de $67,2 \%$ ao final de 5 anos após o transplante. Na avaliação do grupo com doença crônica, a probabilidade de sobrevida dos 30 pacientes foi de $79,8 \%$ com 180 dias e de 76,2\% aos 5 anos após o transplante.Ocorreram sete óbitos, três em decorrência de não funcionamento primário, dois casos de trombose da artéria hepática, um devido a choque séptico e um caso no pós-operatório do tratamento cirúrgico de fístula biliar. As complicações hemodinâmicas ocorreram em $40,5 \%$ dos transplantes de receptores. ${ }^{23,24}$

Dentre as complicações vasculares, a trombose da artéria hepática é grave, levando à perda do enxerto e à indicação de retransplante imediato. É mais frequente na faixa etária pediátrica (7 a 8\%), em grande parte por questões técnicas, como a desproporção do diâmetro entre os vasos do doador e do receptor, com maior risco de estenose e trombose nas anastomoses.

As complicações biliares são, na grande maioria, secundárias a trombose de artéria hepática, e foram observadas em $7,1 \%$ de nossos casos, semelhante ao relatado na literatura (5 a 30\%).

\section{Tabela 3}

Complicações Vasculares Gerais Pós-Transplante Hepático $^{8}$

\begin{tabular}{lcc}
\hline Complicações & $\mathrm{N}^{\circ}$ de casos & $(\%)$ \\
Trombose de Artéria Hepática & 15 & 8,9 \\
Trombose de Veia Porta & 4 & 2,4 \\
Estenose de Veia Porta & 2 & 1,2 \\
Estenose de Veia Cava Inferior & & \\
Infra-Hepática & 2 & 1,2 \\
Estenose de Veia Cava Inferior & & \\
Supra- Hepática & 1 & 0,6 \\
\hline Total & 24 & 14,3 \\
\hline
\end{tabular}


O manejo de complicações biliares está cada vez mais reservado aos grupos de endoscopia com altas taxas de sucesso, ficando reservado a casos selecionados ao tratamento cirúrgico dos pacientes. A via biliar é suscetível a lesões isquêmicas porque seu suprimento vascular origina-se exclusivamente da artéria hepática, sem as vias colaterais que são rompidas durante a captação do enxerto. ${ }^{7}$ Dentre as complicações mais importantes estão as obstruções e as fístulas, ocorrendo em aproximadamente $10 \%$ dos pacientes transplantados segundo a literatura ${ }^{7}$, obstruções essas que podem vir a gerar sinais como icterícia, e as fístulas vindo a gerar hemorragias provenientes do trato digestivo nas fezes.

Sabe-se também, que as complicações biliares associadas a complicações vasculares têm um prognóstico pior e devem ser diagnosticadas precocemente, pois frequentemente necessitam de retransplante. ${ }^{26}$ O tratamento das complicações das vias biliares no transplante depende totalmente de qual é o tipo da complicação.

Da mesma forma nas artérias, principalmente quando o tema da discussão é o transplante pediátrico, é fundamental considerarmos o diâmetro reduzido dos vasos arteriais das crianças em comparação aos adultos, o que facilita muito a formação de trombos nas artérias envolvidas neste procedimento, tal como a artéria hepática.

\section{Conclusão}

Conclui-se que a utilização de enxertos de doadores vivos favorece uma incidência maior de complicações biliares quando comparado aos enxertos de doadores cadáveres, o que determina o inverso das complicações arteriais, sendo essas muito maiores com doadores cadavéricos. As principais complicações no transplante hepático são as complicações biliares, estando a fístula e a estenose biliar em primeiro e em segundo lugar respectivamente, seguido das complicações arteriais que tem a trombose da artéria hepática como a principal complicação desse meio. Tromboses arteriais na fase aguda devem ser manejadas com o re-transplante que representa como fator limitante em nosso meio a escassez de órgãos para a população pediátrica.

\section{ABSTRACT}

Introduction: Liver transplantation greatly improved the survival rate of children and adolescents with terminal liver diseases. Patients, who once had a fatal prognosis, today can be submitted to liver transplantation (LxT), with survival rates of approximately $90 \%$ in one year. The biliary atresia is the main indication in the pediatric population and for those for whom underwent a portoenterostomy and did not obtain good results with it, representing more than $50 \%$ of children under the age of 2 years in Europe and the U.S, transplantation represents the only remaining therapeutic option with good survival benefit. Advances in clinical care and surgical techniques led to improved survival of patients undergoing liver transplantation, but still the arterial and biliary complications represent the Achilles tendon in the development of this procedure. Moreover, the shortage of organs for the pediatric population motivate the development of new modalities of liver grafts, such as reduced liver graft, the split-liver transplantation and more recently the living donors. Despite the improved results, vascular arterial and biliary remain an important cause of morbidity, mortality and graft loss after transplantation. Methods: Survey in Pubmed and Lilacs to gather data related complications of pediatric liver transplantation between 1999 to 2009. Results: In the current study 1485 cases of LxT were performed using cadaveric donors and 505 cases using living donor's grafts were statistically analyzed and data was collected. When analyzing the arterial and biliary complications in pediatric liver transplantation using living donor grafts the rates of biliary fistula was $14.7 \%$, biliary stenosis $3.1 \%$ and arterial thrombosis $3.3 \%$. When analyzing the data regarding cadaveric donors it was observed that the rate of arterial thrombosis was $5.7 \%$, biliary fistula $3.7 \%$ and biliary stenosis (2.5\%). The data was analyzed and compared between the two groups living and cadaveric donors and the results were: biliary fistula $(P=0.0486)^{\star}$, and the biliary stenosis and hepatic artery thrombosis $(P=0.5167)$ and $(P=0.6752)$ respectively. Conclusion: Fistulas and stenosis represent the most frequent biliary complications, especially when using living donors. In the other hand arterial complications are more frequent in the group of cadaveric organ grafts.

Keywords: Liver Transplantation. Pediatrics. Transplantation/complications. Living Donors. Cadaveric donors. Directed Tissue Donation. Cadaver. 


\section{Referências Bibliográficas}

1. Diamond IR, Fecteau A, Millis JM, Losanoff JE, Ng V, Anand $R$ et al. A Report From Studies of Pediatric Liver Transplantation (SPLIT); Ann Surg. 2007; 246: 301-10.

2. Muiesan $P$, Vergani $D$, Mieli-Vergani G. Liver transplantation in children. J Hepatol. 2007;46:340-8.

3. Soares RV , Coelho JCU, Matias JEF, Freitas ACT, Zeni-Neto C, Godoy JL. Anatomia das vias biliares em doadores e receptores de transplante hepático inter vivos. Arq Gastroenterol. 2006; 43: 173-7.

4. Balistreri WF. Transplantation for childhood liver disease: an overview. Liver Transplant Surg. 1998;4:S18-23.

5. Ferreira CT, Vieira SM, Silveira TR. Transplante hepático. J Pediatr. (Rio J.). 2000;76 Suppl 1:S198-208.

6. Busuttil RW, Farmer DG, Yersiz H, Hiatt JR, McDiarmid SV, Goldstein LI. Analysis of long-term outcomes of 3200 liver transplantations over two decades: a single-center experience. Ann Surg. 2005;241:905-16.

7. Freitas ACT, Coelho JCU, Parolin MB, Matias JEF, Neto CZ, Gonçalves CG. Fatores de risco e conduta nas complicações do trato biliar no transplante hepático. Rev Col Bras Cir. 2000; 27: 359-65.

8. Coelho JCU, Matias JEF, Parolin MB, Martins EL, Salvalaggio, PRO, Gonçalves CG. Complicações vasculares pós-transplante hepático. Rev Col Bras Cir. [online]. 2000; 27: 37882.

9. Oliveros F, Santamarýìa M, Gámez M, Murcia J, Leal N, Frauca E, et al. Comparative Study Between Living and Cadaveric Donors in Pediatric Liver Transplantation. Transplant Proc. 37: 3936-8.

10. Borestein S, Diamond IR, Grant DR, Greig PD, Jones N, Vicky $N G$, et al. A. Outcome of pediatric live-donor liver transplantation-the Toronto experience. J Pediatr Surg. 2003:38: 168.

11. Joseph FB, Funaki B, Cronin DC, Yoshida A, Perlman MK, Lorenz J, et al. Long-Term Venous Complications After FullSize and Segmental Pediatric Liver Transplantation Ann Surg. 2002; 236: 658-66.

12. Prabhakaran K, Patankar JZ, Quak SH. Surgical complications and outcome of pediatric liver transplantation: the Singapore experience. Pediatr Surg Int. 2005; 21: 609-14.

13. Otte JB, Reding R, de Ville de Goyet J, Sokal E, Lerut J, Janssen $M$, et al. Experience with living related liver transplantation in 63 children. From the pediatric Liver and intestine transplant program, Saint-Luc University Clinics, Université Catholique de Louvain, Brussels - Belgium. Acta Gastroenterol Belg. 1999; 62:355-62.
14. Broniszczak D, Szymczak M, Kamiñski A, Chyzyñska A, Ismail $\mathrm{H}$, Drewniak T, et al. Vascular Complications After Pediatric Liver Transplantation From the Living Donors. Transplant Proc. 2006; 38:1456-8.

15. Yilmaz A, Arikan C, Tumgor G, Kilic M, Aydogdu S. Vascular complications in living-related and deceased donation pediatric liver transplantation: Single center's experience from Turkey. Pediatr Transplant. 2007;11:160-4.

16. Kelly D. Transplante hepático em crianças. J Pediatr. (Rio J.). 2008: 84: 381-2.

17. Kelly DA. Current results and evolving indications for liver transplantation in children. J Pediatr Gastroenterol Nutr. 1998;27:214-21.

18. Raia S, Nery JR, Mies S. Liver transplantation from live donors. Lancet. 1989;26:497.

19. Strong RW, Lynch SV, Ong TH, Matsunami H, Koido Y, Balderson GA. Successful liver transplantation from a liver donor to her son. N. Engl J Med. 1990; 322:1505-7.

20. Hashikura Y, Makuuchi M, Kawasaki S, Matsunami H, Ikegami T, Nakazawa $Y$, et al. Successful living-related partial liver transplantation to an adult patient. Lancet. 1994;343:12334.

21. Carone E, Chapchap P, Pugliese V, Averbach M, Abdalla R, Saad $\mathrm{R}$. Transplante hepático com doador vivo familiar: técnica operatória no doador. Rev Col Bras Cir. 1997; 24:23540.

22 - Kawashi S, Shimazu M, Wakabayashi G, Hoshino K, Tanabe $\mathrm{M}$, Yoshida $\mathrm{M}$, et al. Biliary complications in adult living donor liver transplantation with duct-to-duct hepaticocholedochostomy or Roux-en-y hepaticojejunostomy biliary reconstruction. Surgery. 2002; 132:48-56.

23. Tannuri U, Velhote MC, Santos MM, Gibelli NE, Ayoub AA, Maksoud-Filho JG, et al. Pediatric liver transplantation: fourteen years experience at the children institute in Sao Paulo, Brazil. Transplant Proc 2004;36:941-2.

24. Mesquita MCO, Ferreira AR, Veloso LF, Roquete MLV, Lima AS, Pimenta JR, et al. Transplante hepático pediátrico: experiência de 10 anos em um único centro no Brasil. J. Pediatr. (Rio J.). 2008;84: 395-402.

25. Chaib E, Ribeiro MAF, Saad WA, Gama-Rodrigues J. The main hepatic anatomic variations for the purpose of split-liver transplantation. Transpl Proc. 2005;37:1063-6.

26. Schlitt HJ, Meier PN, Nashan B et al - Reconstructive surgery for ischemic-type lesions at the bile duct bifurcation after liver transplantation. Ann Surg 1999;229:137-45. 